\title{
The Potential Role of the Edmonton Obesity Staging System in Determining Indications for Bariatric Surgery
}

\author{
Richdeep S. Gill • Shahzeer Karmali • Arya M. Sharma
}

Published online: 15 October 2011

(C) Springer Science+Business Media, LLC 2011

\section{Introduction}

The prevalence of obesity has continued to increase worldwide over the last two decades [1]. Presently, over $20 \%$ of the global population is overweight (BMI $\geq 25 \mathrm{~kg} /$ $\mathrm{m}^{2}$ ), and $10 \%$ is considered clinically obese (BMI $\geq 30 \mathrm{~kg} /$ $\mathrm{m}^{2}$ ) [2]. This equates to approximately 1.5 billion individuals being overweight and 500 million being obese worldwide [3]. These estimates are expected to continue to increase by 2030 to 2.16 billion overweight individuals and 1.12 obese individuals [2]. Similarly, in Canada, approximately $60 \%$ of Canadians are overweight and $24 \%$ are clinically obese [4].

Despite modest weight loss following lifestyle modifications, bariatric surgery is the only evidence-based approach to producing marked and sustainable weight loss in severely obese individuals. Body mass index (BMI) remains the most common measure to estimate adiposity in these individuals, and current guidelines to select appropriateness for bariatric surgery are based on BMI, with Canadian bariatric centers typically offering bariatric surgical management to individuals with BMI $\geq 35 \mathrm{~kg} / \mathrm{m}^{2}$

R. S. Gill

Department of Surgery, University of Alberta,

Edmonton, Alberta, Canada

\section{S. Karmali $(\square)$}

Center for the Advancement of Minimally Invasive Surgery, Royal Alexandria Hospital,

Room 405, Community Services Center, 10240 Kingsway,

Edmonton, Alberta T5H 3V9, Canada

e-mail: shahzeer@ualberta.ca

A. M. Sharma

Department of Medicine, University of Alberta,

Edmonton, Alberta, Canada with obesity-related comorbidities (e.g., type 2 diabetes mellitus) or to any patient with a BMI $\geq 40 \mathrm{~kg} / \mathrm{m}^{2}$. However, BMI alone may not be the optimal measure to guide patient selection for bariatric surgery. Thus, previous studies have suggested that BMI may be a poor prognostic predictor of mortality [5].

Recently, Sharma and Kushner proposed the Edmonton obesity staging system (EOSS) as a more comprehensive measure of obesity-related disease burden and predictor of mortality [6]. In this review, we explore the current evidence in support of EOSS and discuss its potential role to determine indications for bariatric surgery.

\section{Edmonton Obesity Staging System}

The EOSS is a staging system that takes into account severity of comorbidities and functional status in assigning stage (Box 1). Two recent studies in three large and independent populations have compared EOSS to anthropometric measures like BMI or waist circumference as a predictor of mortality. In the first study, Padwal et al. assessed the EOSS retrospectively using a representative cohort population of overweight and obese patients [7]. In this study, individuals with class III obesity (after adjustment for metabolic syndrome or waist line) had virtually no increased mortality risk compared to class II obese individuals (hazard ratio, HR 0.9), while individuals with EOSS 2 or 3 had a 4 to 12-fold greater hazard ratio, respectively, compared to individuals with EOSS 0/1 (Fig. 1). In the second study by Kuk et al., individuals with EOSS stage 2 or 3 had an increased risk of mortality (HR 1.6-1.7) from all causes compared to normal weight individuals [8]. However, neither stage 0 nor 1 was associated with increased mortality risk. 
Box 1. The Edmonton obesity staging system

Stage 0. No apparent risk factors (e.g., blood pressure, serum lipid, and fasting glucose levels within normal range), physical symptoms, psychopathology, functional limitations, and/or impairment of wellbeing related to obesity

Stage 1. Presence of obesity-related subclinical risk factors (e.g., borderline hypertension, impaired fasting glucose levels, elevated levels of liver enzymes), mild physical symptoms (e.g., dyspnea on moderate exertion, occasional aches and pains, fatigue), mild psychopathology, mild functional limitations, and/or mild impairment of well-being

Stage 2. Presence of established obesity-related chronic disease (e.g., hypertension, type 2 diabetes, sleep apnea, osteoarthritis), moderate limitations in activities of daily living, and/or well-being

Stage 3. Established end-organ damage such as myocardial infarction, heart failure, stroke, significant psychopathology, significant functional limitations, and/or impairment of well-being

Stage 4. Severe (potentially end-stage) disabilities from obesity-related chronic diseases, severe disabling psychopathology, severe functional limitations, and/or severe impairment of well-being

\section{The Role of Edmonton Obesity Staging System in Bariatric Surgery}

Currently, bariatric surgery patient selection is commonly based on BMI thresholds alone and a few obesity-related comorbidities. However, patient selection using BMI alone may be criticized as being inaccurate and subjective, since it may not identify those patients that benefit most from bariatric surgery. We suggest that prioritizing patients with increased EOSS scores may identify obese patients with greater mortality risk, since EOSS is better correlated with mortality risk [7]. Determining the urgency for surgical intervention allows for improved prioritization of obese

NHANES III (1988-1994): Class III Obese

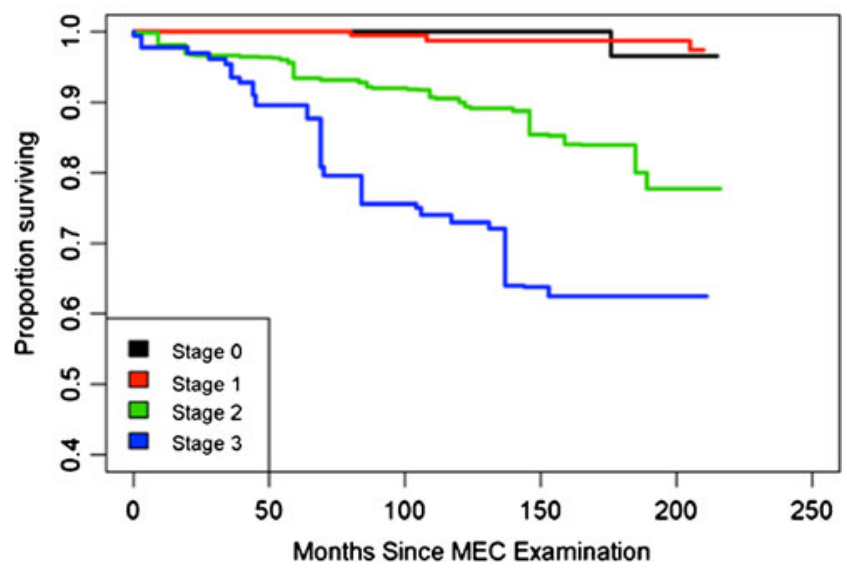

Fig. 1 Edmonton obesity scoring system to predict mortality among patients with class 3 obesity patients. This is highlighted in the study by Kuk et al., which found that a substantial number of patients with class III obesity had minimal mortality risk [8]. With BMI alone used for determining priority for bariatric surgical intervention, many patients with EOSS scores of 2 or 3 may have been overlooked.

It may be argued that bariatric surgery prioritized to EOSS scores 2 and 3, who have increased severity of obesity-related comorbidities, rather than scores $0 / 1$ may miss the opportunity to apply bariatric surgery as a preventative measure. However, in a public-funded health-care system, with limited access and resources, it is prudent to prioritize these resources to those in greatest need. In addition, there is little known about the natural history of obesity, and thus, it remains challenging to predict who will indeed progress to higher EOSS stages and who will remain stable. Therefore, the risk/benefit ratio bariatric surgery in obese individuals with no or minimal obesity-related comorbidities is relatively undetermined. Also, recent evidence suggests that obesity-related comorbidities, such as type 2 diabetes mellitus, may be reversible (in EOSS score $\leq 2$ patients); thus, there may time to delay bariatric surgical management.

Bariatric surgery has been shown to be clinically effective at producing marked weight loss in obese individuals $[9,10]$. An economic evaluation by Picot et al. supported the cost-effectiveness of bariatric surgeryinduced weight loss [11]. However, health technology assessments specify that bariatric surgery is cost-effective in patients with diabetes (EOSS stage 2). In contrast, the cost-effectiveness of bariatric surgery in patients without comorbidities (EOSS <2) is far less clear. Thus, it is likely that a formal health economics analysis based on the EOSS criteria will support the cost-effectiveness of bariatric surgery for EOSS $2 / 3$ patients, with minimal (if any) cost-effectiveness (even in the long-term) in EOSS $0 / 1$ individuals.

\section{Conclusion}

The progressively increasing population of obese individuals is an ongoing global epidemic. Bariatric surgical intervention remains the only evidence-based approach to produce marked sustainable weight loss in severely obese patients. However, with limited health-care resources, it remains an important challenge to determine which patients will benefit most. The EOSS may be an important tool to redefine indications for bariatric surgery in obese individuals. Further research to determine how to incorporate the EOSS into clinical bariatric practice is needed. 
Conflict of Interest The authors RSG, SK, and AMS have no conflict of interests to declare.

\section{References}

1. Finucane MM, Stevens GA, Cowan MJ, et al. National, regional, and global trends in body-mass index since 1980: systematic analysis of health examination surveys and epidemiological studies with 960 country-years and $9 \cdot 1$ million participants. Lancet. 2011;377:557-67.

2. Kelly T, Yang W, Chen CS, et al. Global burden of obesity in 2005 and projections to 2030. Int J Obes. 2008;32:1431-7.

3. WHO. Obesity and overweight. Report no.: fact sheet 311. 2011.

4. Health Canada. Adult obesity prevalence in Canada and the United States. In: Canadian health measures survey. 2011. http:// www.statcan.gc.ca/daily-quotidien/110302/dq110302c-eng.htm. Accessed 15 Aug 2011.
5. Hu FB. Obesity and mortality: watch your waist, not just your weight. Arch Intern Med. 2007;167:875-6.

6. Sharma AM, Kushner RF. A proposed clinical staging system for obesity. Int J Obes. 2009;33:289-95.

7. Padwal RS, Pajewski NM, Allison DB, et al. Using the Edmonton obesity staging system to predict mortality in a populationrepresentative cohort of people with overweight and obesity. CMAJ. 2011;183:1059-66. PMID: 21844111.

8. Kuk JL, Ardern CI, Church TS, et al. Edmonton obesity staging system: association with weight history and mortality risk. Appl Physiol Nutr Metab. 2011;36:570-6.

9. Buchwald H, Avidor Y, Braunwald E, et al. Bariatric surgery: a systematic review and meta-analysis. JAMA. 2004;292:1724-37.

10. Gill RS, Birch DW, Shi X, et al. Sleeve gastrectomy and type 2 diabetes mellitus: a systematic review. SOARD. 2010;6:707-13.

11. Picot J, Jones J, Colquitt JL, et al. The clinical effectiveness and cost-effectiveness of bariatric (weight loss) surgery for obesity: a systematic review and economic evaluation. Health Tech Assess. 2009;13:1-190 